1991-01-01

\title{
A median-filter-based ambiguity removal algorithm for NSCAT
}

David G. Long

david_long@byu.edu

Scott J. Shaffer

R. Scott Dunbar

S. Vincent Hsiao

Follow this and additional works at: https://scholarsarchive.byu.edu/facpub

Part of the Electrical and Computer Engineering Commons

\section{Original Publication Citation}

Shaffer, S. J., et al. "A Median-Filter-Based Ambiguity Removal Algorithm for NSCAT."

Geoscience and Remote Sensing, IEEE Transactions on 29.1 (1991): 167-74

\section{BYU ScholarsArchive Citation}

Long, David G.; Shaffer, Scott J.; Dunbar, R. Scott; and Hsiao, S. Vincent, "A median-filter-based ambiguity removal algorithm for NSCAT" (1991). Faculty Publications. 726.

https://scholarsarchive.byu.edu/facpub/726

This Peer-Reviewed Article is brought to you for free and open access by BYU ScholarsArchive. It has been accepted for inclusion in Faculty Publications by an authorized administrator of BYU ScholarsArchive. For more information, please contact ellen_amatangelo@byu.edu. 


\title{
A Median-Filter-Based Ambiguity Removal Algorithm For NSCAT
}

\author{
Scott J. Shaffer, Member, IEEE, R. Scott Dunbar, S. Vincent Hsiao, and David G. Long, Member, IEEE
}

\begin{abstract}
The NASA Scatterometer, NSCAT, is an active spaceborne radar designed to measure the normalized radar backscatter coefficient $\sigma_{0}$ of the ocean surface. These measurements can, in turn, be used to infer the surface vector wind over the ocean using a geophysical model function. Because of the nature of the model function, several ambiguous wind vectors result. A process commonly known as "dealiasing" or ambiguity removal must be used to select the "best" wind vector from the set of ambiguous wind vectors. An automated, median-filter-based ambiguity removal algorithm which requires only the scatterometer measurements will be used by the NSCAT ground data-processing system. The algorithm incorporates a number of selectable parameters such as window size, mode, and likelihood weighting which can be adjusted to optimize algorithm performance. This paper describes the baseline NSCAT ambiguity removal algorithm and the method used to select the set of optimum parameter values. An extensive simulation of the NSCAT instrument and ground data processor provides a means of testing the resulting "tuned" algorithm. This simulation generates the ambiguous wind-field vectors expected from the instrument as it orbits over a set of realistic mesoscale wind fields. The ambiguous wind field is then dealiased using the medianfilter-based ambiguity removal algorithm. Performance is measured by comparison of the selected wind fields with the "true" wind fields. Results have shown that this median-filter-based ambiguity removal algorithm satisfies NSCAT mission requirements, and it therefore has been incorporated into the baseline geophysical data-processing system for NSCAT.
\end{abstract}

\section{INTRODUCTION}

$\mathrm{T}$ HE feasibility of spaceborne scatterometers to make estimates of both wind speed and direction over the ocean was demonstrated by the Seasat-A scatterometer [5]. A scatterometer measures the normalized radar backscatter coefficient $\sigma_{0}$ of the ocean surface. These measurements can then be used to estimate vector surface wind using a geophysical model function and a wind retrieval algorithm. The NASA scatterometer, NSCAT, is scheduled to fly in the mid-1990's [9]. The $\sigma_{0}$ data will be processed to vector wind estimates at $50-\mathrm{km}$ resolution using a point-wise Maximum Likelihood (ML) technique [3]. Because of the harmonic dependence of $\sigma_{0}$ on wind direction, though, the retrieval technique is unable to uniquely resolve the wind vector. A set of 2 to 6 possible wind vectors known as ambiguities are determined [2]. Associated with each ambiguity is a likelihood value which may be used to order the ambiguities. The NSCAT ambiguity removal algorithm, employing only scatterometer-derived data, will be used to select one wind vector from the set of ambiguities that, when successful, is the closest vector to the true wind for each wind resolution cell.

Manuscript received October 10, 1989; revised February 22, 1990. The work described in this paper was performed at the Jet Propulsion Laboratory, California Institute of Technology, under contract with the National Aeronautics and Space Administration.

The authors are with the Radar Science and Engineering Section, Jet Propulsion Laboratory, California Institute of Technology, 4800 Oak Grove Drive, Pasadena, CA 91109

IEEE Log Number 9036092
The problem of ambiguity removal for scatterometer-derived wind vectors has been studied extensively for Seasat SASS [11]. The advantage of a third scatterometer beam for ambiguity removal was pointed out by Schroeder [10] for the NOSS scatterometer design, which was the forerunner of the NSCAT 3-antenna, 4-beam design. Ambiguity removal algorithm development for NSCAT, since 1986 , has mainly concentrated on the median filter concept.

This paper describes the NSCAT ambiguity removal algorithm and the process used to optimize the adjustable parameters (tuning) and to evaluate the algorithm's performance. The wind field and instrument simulations used to provide the test input data for this process are also described, and the methods used to evaluate algorithm performance are developed. Finally, the results of tuning the algorithm parameters and the subsequent performance tests are presented.

\section{Algorithm Description}

The ambiguity removal algorithm employed in this investigation uses a modified median filter technique to select a unique wind vector out of a set of ambiguous wind vectors at each wind vector cell (WVC). Median filter techniques were first introduced in 1974 to filter time-series data, and were later applied to image enhancement. The median of a set of $N$ values is defined for $N$ (odd) as the $(N+1) / 2$-th largest number; i.e., there is an equal number of values greater and lesser in magnitude than the median. Because the selection of a median is determined only by the order of numbers, the median is not affected by extremely large or small values in the data. The advantage of a median filter is that it eliminates isolated impulses while preserving edges. On the contrary, a linear filter such as a mean filter or a Hamming window filter smooths, rather than eliminates, impulses and smears edges.

The conventional definition of the median can only be applied to scalar data in which the ordering of the values is obvious. The pixel brightness levels in an image is an example. For circular data (such as directions) or vector data the conventional definition of median fails because the order of numbers cannot be specified, and the median itself can be ambiguous. One method given by Mardia [8] defines the median of directional data to be the direction $\theta$, such that an equal number of data exist in the half circles $\left(\theta, \theta+180^{\circ}\right)$ and $\left(\theta-180^{\circ}, \theta\right)$. This definition is not only cumbersome for computation, but also gives multiple solutions. An alternative definition, also due to Mardia, is that the circular mean deviation is a minimum when measured from the median direction, i.e., for circular data $a(1)$, $a(2), \cdots, a(N)$, and that the median is the value $a(m)$ which minimizes the function:

$$
S(m)=\sum_{i=1}^{N}|a(m)-a(i)|, \quad 1 \leq m \leq N
$$


Here the absolute deviations are defined as positive angles between $0^{\circ}$ and $180^{\circ}$. This formulation is much simpler from a computational standpoint than the conventional definition. For multidimensional data such as vectors and complex numbers, it is even more difficult to define the median. However, the minimum deviation approach can be extended to define the median of multidimensional data. For vector data $V(i)$, the median can be defined as the vector $V(m)$ which minimizes $S(m)$, where

$$
S(m)=\sum_{i=1}^{N}\|V(m)-V(i)\| \quad \text { and } \quad 1 \leq m \leq N .
$$

The vector norm formulation for the vector median as defined here behaves similarly to the median of scalar and circular data in that it is also unaffected by extreme values in the data. The minimum deviation approach given by (1), and its extension in (2), form the basis for our subsequent development of a windvector ambiguity removal algorithm.

A median-filter based on (1) or (2) is implemented by constructing a fixed-length data window which is passed over the data. At each step in the filtering process, the computed median of the values in the window replaces the value at the center of the window interval. Applying a one-dimensional filter of length $N$ to a sequence of vector data $\left\{\boldsymbol{A}_{i}\right\}$, we may rewrite (2) in the form of the "error" function $E_{i}$ :

$$
E_{i}=\sum_{m=i-h}^{i+h} W_{m^{\prime}}\left\|\boldsymbol{A}_{i}-\boldsymbol{A}_{m}\right\|
$$

where $h=(N-1) / 2$ is the half-width of the window centered on $A_{i}$, and $W_{m^{\prime}}$ is an arbitrary window weighting function that determines the contribution of each term relative to the centra value $\left(m^{\prime}=m-i\right)$. In this case, we seek a replacement for $\boldsymbol{A}$ from the set $\left\{\boldsymbol{A}_{m}\right\}$ that minimizes $E_{i}$. The directions-only filter is derived analogously to (3), except that the vector norm $\| \boldsymbol{A}$ $-\boldsymbol{A}_{m} \|$ is replaced by the direction norm:

$$
\left|\theta_{i}-\theta_{m}\right|=\cos ^{-1}\left[\frac{\boldsymbol{A}_{i} \cdot \boldsymbol{A}_{m}}{\left|\boldsymbol{A}_{i}\right|\left|\boldsymbol{A}_{m}\right|}\right] .
$$

The extension of (3) to create a median filter of a two-dimensional vector field $\left\{\boldsymbol{A}_{i j}\right\}$ is trivial:

$$
E_{i j}=\sum_{m=i-h}^{i+h} \sum_{n=j-h}^{j+h} W_{m^{\prime} n^{\prime}}\left\|\boldsymbol{A}_{i j}-\boldsymbol{A}_{m n}\right\|
$$

where $\boldsymbol{A}_{i j}$ is the vector at the center of the $N \times N$ window ( $N$ odd ), $h$ is defined as for (3), $W_{m^{\prime} n^{\prime}}$ is the window weight function (with $m^{\prime}=m-i$ and $n^{\prime}=n-j$ ), and we seek to replace $\boldsymbol{A}_{i j}$ with a vector from $\left\{\boldsymbol{A}_{m n}\right\}$ that minimizes $E_{i j}$. Extension of the direction-only filter to two dimensions is accomplished by replacing the vectors in (4) with doubly indexed vectors in (4) and substituting for the vector norm in (5).

The final extension required for the wind-vector ambiguity problem is to consider the two-dimensional wind-vector field $\left\{\boldsymbol{A}_{i j}^{k}\right\}$, where each grid point $(i, j)$ on the field has a set of $k$ ambiguous vectors. Associated with each wind vector is a likelihood $L_{i j}^{k}$ assigned by the wind-retrieval algorithm. Let us assume, for convenience, that the vectors have been sorted in descending likelihood order (i.e., $\boldsymbol{A}_{i j}^{\prime}$ is the highest likelihood). We may also weight each ambiguity by a simple power function of its likelihood value $\left(L_{i j}^{k}\right)^{p}$, where $p \geq 0$. Let $\boldsymbol{U}_{i j}$ be an array of vectors on the $(i, j)$ grid selected from the $\boldsymbol{A}_{i j}^{k}$ ambiguities, initialized with the set of highest-likelihood vectors:

$$
\boldsymbol{U}_{i j}=\boldsymbol{A}_{i j}^{l}, \quad \text { for all } i, j \text {. }
$$

We may now write our formulae for the median filters as: Direction-Only Median

$$
E_{i j}^{k}=\frac{1}{\left(L_{i j}^{k}\right)^{p}} \sum_{m=i-h}^{i+h} \sum_{n=j-h}^{j+h} W_{m^{\prime} n^{\prime}} \cos ^{-1}\left[\frac{A_{i j}^{k} \cdot U_{m n}}{\left|A_{i j}^{k}\right|\left|U_{m n}\right|}\right]
$$

Vector Median

$$
E_{i j}^{k}=\frac{1}{\left(L_{i j}^{k}\right)^{p}} \sum_{m=i-h}^{i+h} \sum_{n=j-h}^{j+h} W_{m^{\prime} n^{\prime}}\left\|A_{i j}^{k}-U_{m n}\right\| .
$$

The initial field $\boldsymbol{U}_{i j}$ selected from the field of ambiguities is compared via (7) or (8) with each member of the set $\left\{A_{i j}^{k}\right\}$ in the center of the filter window, computing a set of values $\left\{E_{i j}^{k}\right\}$ of the filter function. The ambiguity that minimizes the filter function is selected to be $\left.U_{i j}^{*}\right\}$, the new array of selected vectors which replaces $U_{i j}$ for the next iteration. The filtering/selection process continues at each grid point $(i, j)$ in the array to create $\boldsymbol{U}_{i j}^{*}$. Then $\boldsymbol{U}_{i j}$ is replaced by $\boldsymbol{U}_{i j}^{*}$, and the whole process is repeated to select a new $U_{i j}^{*}$. This iterative process continues until $U_{i j}^{*}=U_{i j}$. Note that two copies of $\boldsymbol{U}_{i j}$ must be maintained to ensure stability and convergence of the process.

An advantageous feature of surface wind-vector fields is that they are relatively smooth and continuous. There are significant discontinuities such as fronts and convergence zones, as well as cyclonic structures, that must be preserved by the ambiguity removal algorithm. The median filter is thus well suited to the problem in that it will eliminate random selection errors, while simultaneously maintaining those large-scale discontinuities that are of key interest. However, if the ambiguity removal algorithm based on the foregoing formulation is to succeed, it is necessary that the wind-retrieval algorithm produce an initial wind field in which the true features are dominant.

The NSCAT maximum-likelihood wind-retrieval algorithm computes a set of wind vectors ranked by their relative likelihoods for each wind-vector cell (WVC) [3]. The highest-ranked solution is referred to as the "most likely" wind vector. The solution closest to the true wind is called the "closest" solution, and all the wind-vector solutions found in each WVC are referred to as "ambiguities." Based on simulated NSCAT data, there are usually two to six ambiguities per WVC. Typically, about $90 \%$ of the two "most likely" (i.e., highest value of likelihood) ambiguities are either the closest vectors, or are about $180^{\circ}$ opposite to the closest vector. For NSCAT, the solution with the highest likelihood is the closest vector about $60 \%$ of the time. The objective of the ambiguity removal algorithm is to choose the closest solution from the list of ranked ambiguities, which is equivalent to detecting cases where the most likely ambiguity is not the closest vector and then selecting the correct solution.

The median-filter ambiguity removal algorithm was chosen for use by NSCAT because it requires no auxiliary data (in situ data, numerical weather models, etc. ) and because of its simplicity, tunability, and overall performance. The vector median computation given in (8) retains the desirable properties of a median filter. The additional weighting parameters $\left[W_{m r^{\prime} n^{\prime}}\right.$ and $\left.\left(L_{i j}^{k}\right)^{\prime}\right]$ have been introduced to permit algorithm tuning and performance enhancement.

The four adjustable parameters which permit algorithm tuning are: Mode (direction $(0)$ or vector median $(1)$ ), window size $(N)$, likelihood weight exponent $(p)$, and window weight $\left(W_{m^{\prime} n^{\prime}}\right)$. The mode controls whether wind speed is included in the calculation of the error function; $N$ can range between 3 and 11 WVC's on a side; $W_{m^{\prime} n}$ controls the relative contribution of 
each vector in the window; and $p$ determines the advantage given to vectors with a higher retrieval algorithm likelihood.

\section{Algorithm Performance}

The performance of any given ambiguity removal algorithm is ultimately determined by its ability to faithfully reproduce the intrinsic features of the observed wind field. A robust algorithm must be capable of handling the full range of possible situations. In this section we discuss the principal factors that affect algorithm performance and introduce several metrics with which to analyze and optimize the median-filter algorithm.

External factors affecting the performance of any ambiguity removal algorithm are attributable to either the geophysical model function or the scatterometer design. The azimuthal modulation of the backscatter/wind model function, or upwind/ downwind asymmetry, exhibited by the model function affects the ability of the retrieval algorithm to distinguish the relative likelihood of the principal ambiguities, which are typically $180^{\circ}$. The degree of upwind/downwind asymmetry of a given model varies with the incidence angle, polarization, and wind speed.

The relative amount of noise as measured by the signal-tonoise ratio (SNR) in the backscatter measurements affects not only the wind vector solutions but also their assigned likelihoods. The numbers, azimuthal arrangement, and polarizations of the scatterometer antenna beams also affect the wind retrieval [7]. On NSCAT, the introduction of a dual-polarization middle beam in addition to the two orthogonal fore/aft beams (see Fig. 1) is intended to improve the ability of the retrieval algorithm to distinguish the correct wind vector.

This "native" ability of the instrument/wind retrieval algorithm to find the correct solution is referred to as "instrument skill." Instrument skill is defined as the percentage of WVC's for which the highest-likelihood wind vector is also the vector closest to the true wind. An instrument skill of $100 \%$ would indicate a "perfect" retrieval algorithm; i.e., no ambiguity removal required. For NSCAT, the averge instrument skill is typically of the order of $60 \%$. An analogous statistic for the ambiguity removal algorithm is called the "ambiguity removal skill." We define ambiguity removal skill as the percentage of WVC's for which the wind vector selected by the ambiguity removal algorithm is also closest to the true wind.

In general, good ambiguity removal skill can be achieved when the average instrument skill is high. However, the average instrument skill alone does not provide sufficiently detailed information. The instrument skill can vary systematically in different regions of the measurement swath, depending on measurement SNR and relative azimuth of the antenna beams to the local wind direction. This effect is referred to as "local instrument skill"; that is, the skill of the wind-retrieval algorithm on small spatial scales. Local skill can be quite low for far-swath cells and low-wind speeds (due to low SNR) and at particular wind directions where the wind-retrieval algorithm cannot distinguish (or reverses) the relative likelihoods of the two principal ambiguities. This causes ambiguity selection errors to appear in clusters rather than being randomly distributed. Sufficiently large regions of low skill can cause ambiguity selection errors to propagate, depending on the algorithm used. Based on simulated NSCAT data, high algorithm skills ( $>96 \%$ ) with randomly distributed errors can usually be attained when regions of low instrument skill are much smaller than the medianfilter window size.

Two performance metrics were adopted to select the optimum

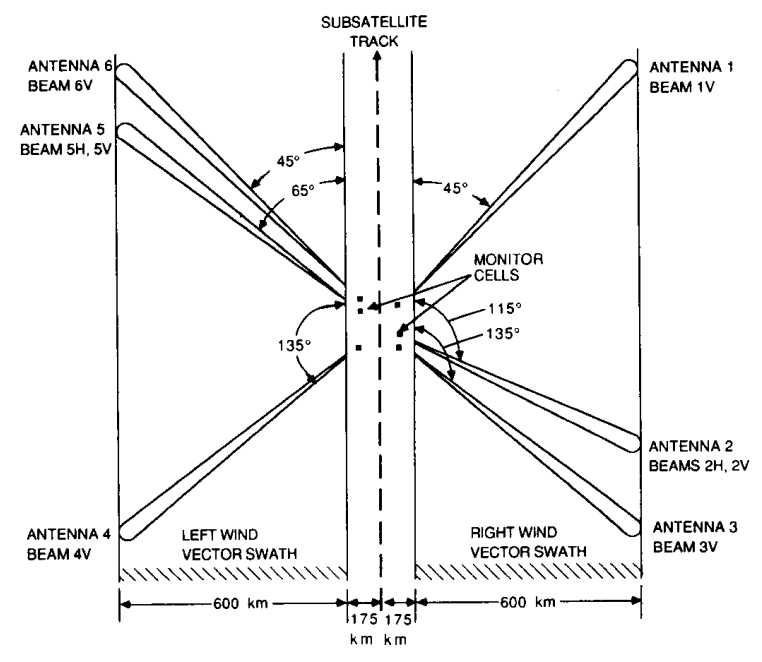

Fig. 1. The NSCAT antenna beam and swath configuration.

set of ambiguity removal parameters: The ambiguity removal (algorithm) skill defined above, and a 12 by 12 WVC "clumpiness" metric. Clumpiness is the tendency of ambiguity selection errors to occur in the proximity of other ambiguity errors. It is defined as the percentage of 12 by 12 contiguous WVC regions having greater than $85 \%$ successful selection. This metric increases as the ambiguity removal errors become more randomly distributed. The NSCAT science requirements state that the algorithm skill must be greater than $96 \%$ and the clumpiness metric be greater than $98 \%$ in regions of the swath where the true wind speed is between 3 and $30 \mathrm{~m} / \mathrm{s}$ [7]. To conform with these requirements, both performance measurements are only calculated in this wind speed region.

\section{Algorithm Testing Procedures}

Our approach to testing the NSCAT ambiguity removal algorithm consists of two stages: Parameter optimization (tuning) and algorithm evaluation. The goal of ambiguity removal tuning is to select the set of parameters which maximizes the algorithm performance. The optimized algorithm is then tested to determine its strengths and weaknesses.

An extensive simulation of the NSCAT instrument and realistic wind fields, described in Section V, provided the input datasets for these tests. The obvious advantage to using a simulation is that the true wind field is available for comparison with the results of the tests. The winds fields from which the instrument data were simulated were divided into two separate groups-a test group and a withheld group. The test group was used to optimize the ambiguity removal algorithm, while the withheld group was used for the performance evaluation of the algorithm. The simulated instrument data was processed to backscatter measurements and then to ambiguous, ranked wind vectors.

In the tuning stage, over 100 different input parameter configurations for the algorithm were used, spanning most degrees of algorithm freedom: Mode, window size, location weight, and likelihood weight. Each configuration of the algorithm was run to dealias the six ambiguous wind files in the test group. For each run, the algorithm skill and clumpiness metrics were computed to characterize the parameter configuration. In addition, 


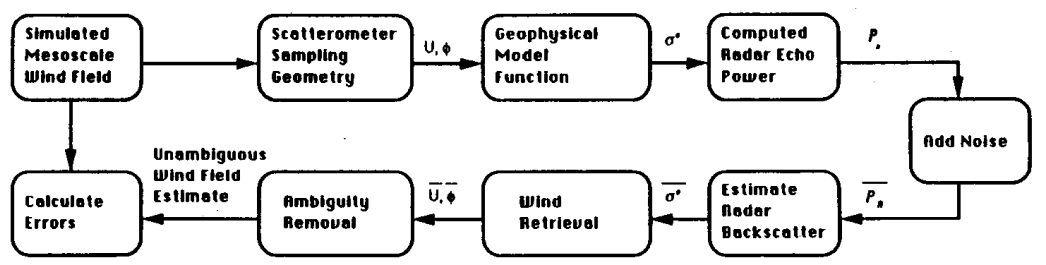

Fig. 2. Flow diagram of the NSCAT instrument/wind field simulation that was used to provide realistic scatterometer data for testing the ambiguity removal algorithm.

spatial plots of the ambiguity errors and distributions of the errors as a function of wind speed and swath location were made as aids to visualization. The performance metrics were averaged over the six wind files in the test group, providing a measure for ranking the sets of input parameters. The results of the tuning stage are discussed in Section VI.

Having optimized the algorithm to account for "typical" wind fields and instrument and model function factors, the algorithm is evaluated against a further set of wind fields (the withheld set) to test its robustness-the algorithm versus the winds. Again, the skill and clumpiness metrics are averaged over the six wind fields to measure performance. Any significant deviation in the measured performance from that found in the tuning stage would indicate sensitivity to the peculiarities of the wind vector fields, an undesirable result.

\section{InStrument/Wind Field Simulation}

The spaceborne NSCAT instrument uses 6 fan-beam antennas to provide multiple azimuth angle observations of the normalized radar backscatter $\left(\sigma_{0}\right)$ of each resolution element (Fig. 1). The $\sigma_{0}$ measurement cells have a resolution of $25 \mathrm{~km}$, with 24 on each side of the spacecraft. The wind measurements are retrieved at $50-\mathrm{km}$ resolution (12 on each side) with $16 \sigma_{0}$ 's per WVC.

We have developed an end-to-end system simulation of NSCAT, including both instrument and ground-processing systems. In this simulation the scatterometer is "flown" over realistic wind fields. The changing orbital geometry is included. Monte Carlo techniques are used to generate $\sigma_{0}$ measurement noise, geophysical modeling error, and $\sigma_{0}$ retrieval error [2]. All noise components are assumed to be Gaussian. The $\sigma_{0}$ measurement noise, which is a function of SNR, is based on the measurement geometry and NSCAT hardware design [6]. The SNR can range from a low of $-20 \mathrm{~dB}$ to a high of $18 \mathrm{~dB} \mathrm{de}$ pending on the wind-dependent $\sigma_{0}$ and the measurement cell location. The geophysical modeling error reflects the uncertainty in the SASS I model function relating $\sigma_{0}$ and the surface wind vector. Following earlier work, a value of $0.7 \mathrm{~dB}$ has been used for the model function uncertainty [2]. The retrieval error arises from the uncertainty in the parameters of the radar equation such as the slant range, antenna gain, and cell area used to compute $\sigma_{0}$ from the instrument power measurement. Uncertainty values are based on the instrument calibration accuracy, the antenna pointing uncertainty budget, and the orbit determination budget. The retrieval error amounts to about $0.7 \mathrm{~dB}$, depending on the orbit and measurement cell location.

As shown in Fig. 2, at each step of the simulation each of the measurement cells is first located in latitude and longitude. After conversion to swath coordinates (along-/cross-track), the mesoscale wind vector ("true" wind) at that location is assigned to the cell. The wind speed and direction, along with the

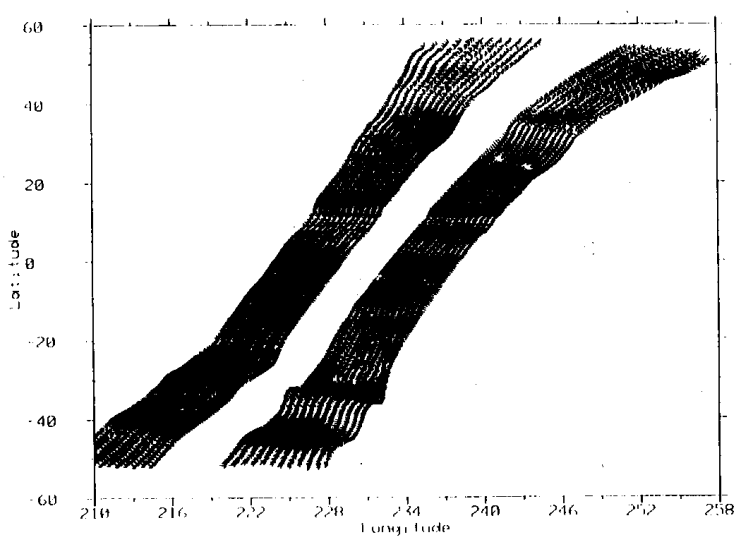

Fig. 3. An example of a mesoscale wind field mapped onto the NSCAT wind measurement swath. The wind vectors are shown here at $100-\mathrm{km}$ resolution (every other vector) for clarity; the winds are converted to simulated backscatter measurements at $25-\mathrm{km}$ resolution, and retrieved at 50 $\mathrm{km}$ resolution.

local incidence angle and beam polarization, are input to the geophysical model function to produce a "true" $\sigma_{0}$. This true $\sigma_{0}$ is converted to a received power via the radar equation. Noise is then added to produce a simulated scatterometer power measurement. These steps are repeated for all $\sigma_{0}$ cells on the swath. In the ground-processing simulation, the "measured" $\sigma_{0}$ 's are then recovered and assigned to 50-km wind vector cells. A point-wise maximum-likelihood wind-retrieval algorithm [3] is used to generate ambiguous winds.

As input to this system simulation, high-resolution mesoscale surface wind fields are required. Since little conventional mesoscale wind field data over the ocean are available, simulated wind fields were generated. As described by Bevan and Freilich [1], original ECMWF surface wind fields at $1.875^{\circ}$ resolution were interpolated to $10 \mathrm{~km}$ and nondivergent small-scale variability with a $a k^{-2}$ spectrum [4] was added. For a given 2000 $\times 2000 \mathrm{~km}$ region, the value of " $a$ " was selected to be consistent with the spectrum within the region. Twelve wind fields were selected to span a wide range of meteorological conditions, including sharp fronts and small-scale cyclones with wind speeds between 0 and $30 \mathrm{~m} / \mathrm{s}$. An example of one mesoscale field used in this study is plotted in Fig. 3.

\section{Parameter Optimization}

The variation in ambiguity removal performance observed in this part of the study indicates that algorithm tuning can have a small but significant effect. About $75 \%$ of the cases achieved an ambiguity removal skill between 94 and $97 \%$. Thus the gain in algorithm skill achievable by adjustment of the parameters is 


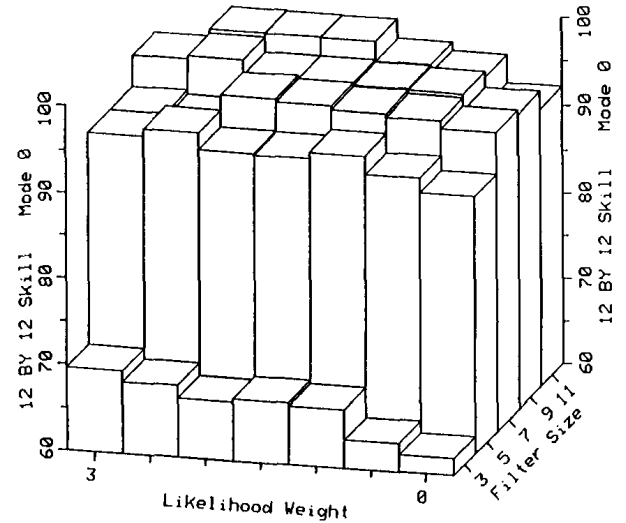

(a)

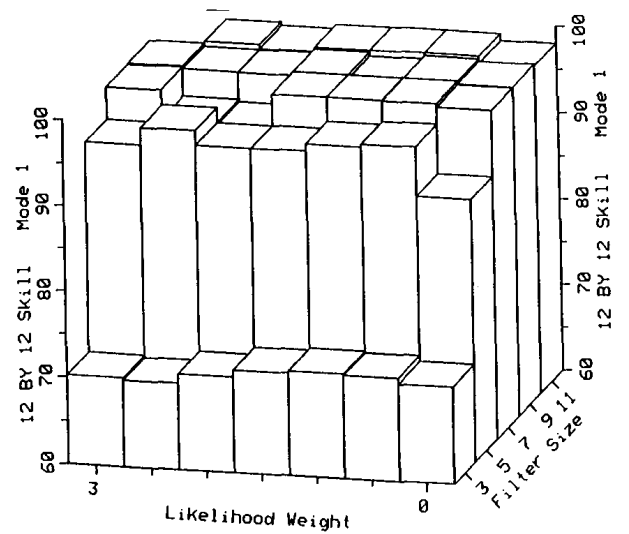

(b)

Fig. 4. The algorithm skill is plotted as a function of filter size and likelihood weight for both mode 0 (a), and mode 1 (b).

small. The 12 by 12 metric exhibited similar behavior. Over $73 \%$ of the filters evaluated achieved better than $95 \%$ of the 12 by 12 contiguous regions with over $85 \%$ successful selection. Forty-six percent of the filters achieved better than the required $98 \%$. Therefore the improvement in the 12 by 12 metric achievable by tuning the algorithm parameters is also small.

One of the most prominent features is the dependence on the algorithm mode. The "mode" parameter selects between one of two distinct median-filter algorithms. In every case where two configurations differ only by mode, the configuration using the vector median algorithm (mode 1) has a higher algorithm skill; hence the vector error function performs better than the direction error function. The superiority of the vector median formulation (equation (8)) was recognized at an early stage in the tuning process.

The median-filter algorithm also exhibits a strong dependence on window size. The filter window size sets the number of wind cells adjacent to a particular cell that are utilized in the ambiguity removal process. The window dimensions must be odd integers so as to ensure that the particular WVC under consideration is in the center of the window. In these tests the windows were not restricted to being square, but it was found that square filters performed better than rectangular filters. The algorithm performance peaks at 7 by 7 , although the difference

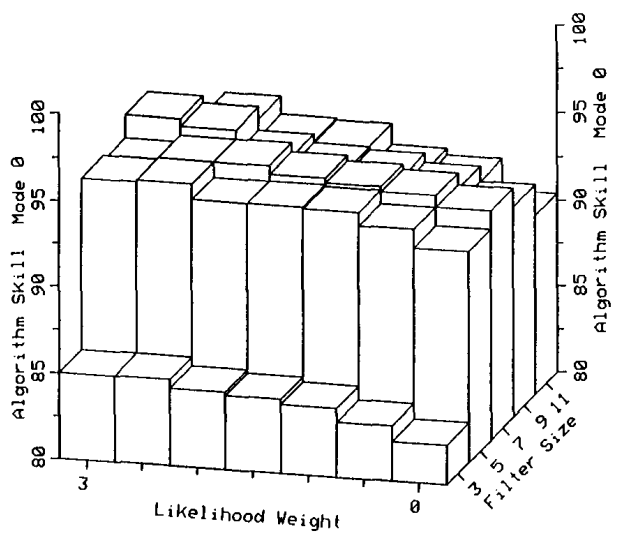

(a)

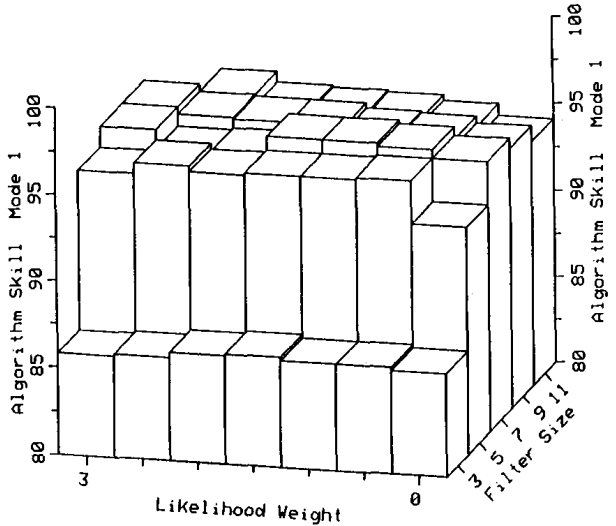

(b)

Fig. 5. The 12 by 12 clumpiness metric is plotted as a function of filter size and likelihood weight for both mode 0 (a), and mode 1 (b).

between 5,7 , and 9 is quite small. The algorithm skill drops substantially when the window size is reduced to 3 by 3 WVC's.

The results also display a slight dependence upon the likelihood weighting. The algorithm skill for both modes 0 and 1 increases as the likelihood weight increases between 0 and 2 . The skill for mode 1 drops as the likelihood weight increases from 2 to 3 . Mode 0 increases slightly over the same range, but the increase is insignificant. Fig. 4 summarizes this observed relationship between mode, window size, likelihood weight, and the average algorithm skill for uniformly weighted configurations over the test group of wind files. A comparison has also been made using the 12 by 12 metric with similar results (see Fig. 5).

With the exception of configurations using mode 0 or a window size, $N=3$, the algorithm performance is quite good and fairly uniform, making the selection of a single "optimum" configuration difficult. Based on Figs. 4 and 5, the optimum set of ambiguity removal algorithm parameters has been chosen as follows: Mode $=1$ (vector median error function); filter size $=7$ by $7 \mathrm{WVC}$; filter shape = square, uniform weight; and likelihood weight $=2$ (advantage to "most likely" ambiguities). With these values the median-based ambiguity removal algorithm achieves an algorithm skill of $96.7 \%$ and a 12 by 12 metric of $98.7 \%$ over the test group. 

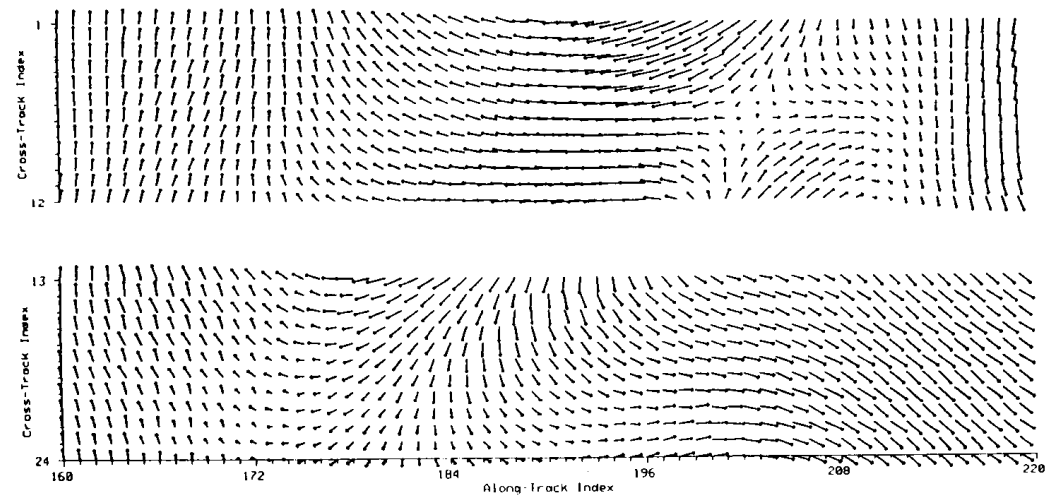

(a)
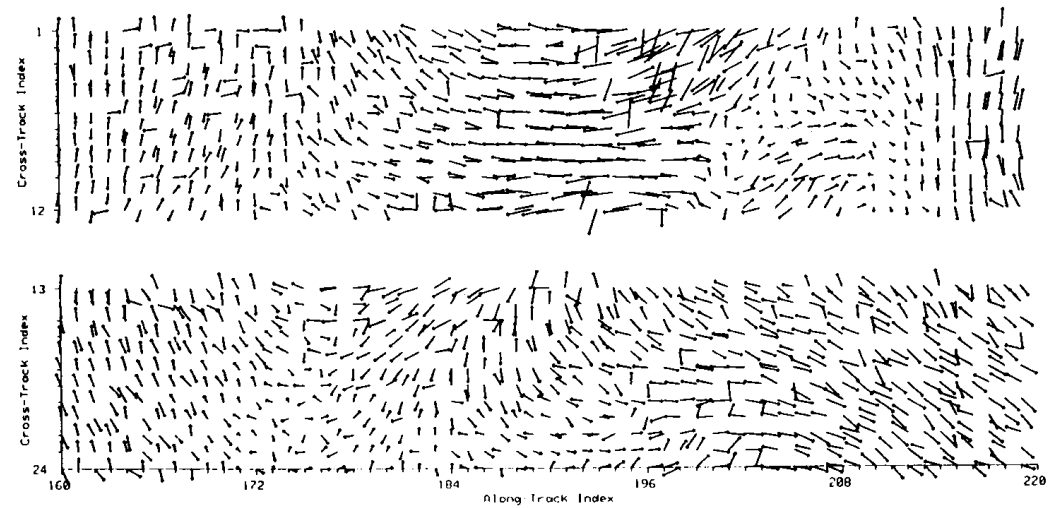

(b)
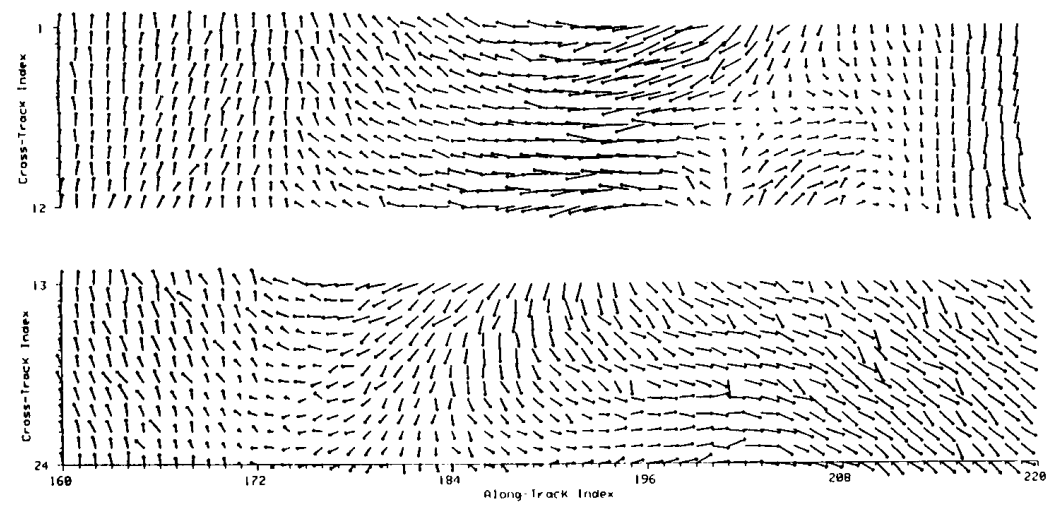

(c)

Fig. 6. A magnified example of one test wind field, showing: (a) The starting "true" wind: (b) the field of highest-likelihood ambiguities alter wind retrieval; (c) the field of vectors selected by the median-filter ambiguity removal algorithm.

\begin{abstract}
Although several configurations utilizing nonuniform location weighting were also tested, it is clear that any improvement in performance due to location weighting will be small. Detailed analysis of nonuniform configuration performance was complicated by the large number of possible weighting gradients.
\end{abstract}

\section{Algorithm Performance Evaluation}

To validate the selection of an optimum set of algorithm parameters, the test was repeated using the withheld group of wind fields. Although the average performance of all algorithm configurations was slightly lower for the withheld data set, the drop 

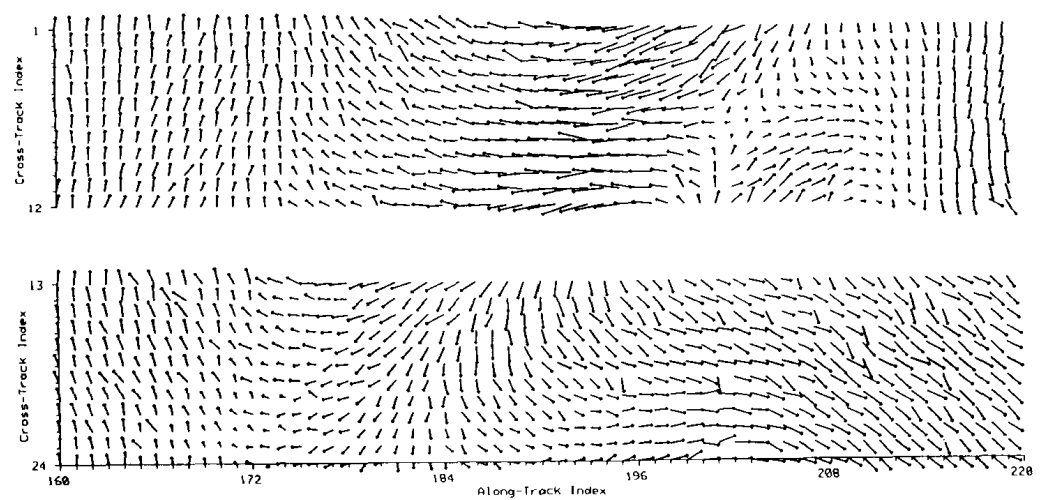

(d)

Fig. 6. (continued). (d) The field of ambiguities "closest" to the "true"' winds (i.e., that should have been selected)

TABLE I

Median-Based Ambiguity Removal Algorithm Metric Performance

\begin{tabular}{lcc}
\hline Wind Files & $\begin{array}{c}\text { Algorithm } \\
\text { Skill }\end{array}$ & $\begin{array}{c}12 \text { by } 12 \\
\text { Metric }\end{array}$ \\
\hline Test Set & $96.7 \%$ & $98.69 \%$ \\
Withheld Set & $96.0 \%$ & $98.07 \%$ \\
Combined & $96.4 \%$ & $98.38 \%$ \\
\hline
\end{tabular}

was not significant. Several of the highest-ranking filters from the tuning stage were also re-tested on the withheld set and their results were ranked against the new baseline. The optimum parameters configuration selected above ranked 4th using the withheld group of wind fields. The difference in algorithm skill, however, between the tuned optimum and the highest ranked configuration for the withheld group was only $0.3 \%$. Because the individual wind files which went into the average algorithm skill may have had different average instrument skills, a high standard deviation would not have been unexpected. However, the standard deviation in the algorithm performance averaged over the six withheld wind fields is approximately $2 \%$, making this difference insignificant. When the statistics from both wind field sets were combined, the optimum set of algorithm parameters ranked the highest. The performance of both metrics for the optimum algorithm configuration is summarized in Table I.

Fig. 6(a)-(d) demonstrates the characteristics of the true wind field, the "most likely" wind field, the selected field after ambiguity removal, and the field of "closest" wind vectors (found by direct comparison of the ambiguities with the true field) for a portion of one of the test wind files. Differences between the "selected" (Fig. 6(c)) and "closest" (Fig. 6(d)) fields are interpreted as algorithm errors. The difference between the "closest" and "true" fields are due to noise added in the simulation.

\section{Conclusion}

The median-filter-based ambiguity removal algorithm is a very effective method of selecting the closest vector to the true wind field from a set of ambiguities. It is a simple algorithm to implement and performs well over a large variety of wind fields.
An optimum configuration for the ambiguity removal algorithm has been selected. The configuration consists of a 7 by 7 square filter with all locations within the filter weighted equally and an advantage given to ambiguities selected as the most likely wind vector by the wind-retrieval algorithm. The algorithm skill is $96.7 \%$, with $98.69 \%$ of the 12 by 12 contiguous regions having greater than $85 \%$ successful selection. A comparative performance study of several ambiguity removal algorithms, including the median-filter algorithm presented here, is planned. This algorithm, to be used for NSCAT, can be applied as well to other three-beam scatterometers such as the ERS-1.

\section{REFERENCES}

[1] R. Bevan and M. Freilich, "Wind fields for Big Sim," JPL, Pasadena, CA, JPL Memo. NSCAT/MHF 87-15, Oct. 1, 1987

[2] C.-Y. Chi, R. Aroian, and F. Li, "Simulation studies for the NASA scatterometer on NROSS,' in Proc. Int. Geosci. Remote Sensing Symp., Sept. 1986, pp. 1673-1676.

[3] C.-Y. Chi and F. K. Li, "A comparative study of several wind estimation algorithms for spaceborne scatterometers," IEEE Trans. Geosci. Remote Sensing, vol. 26, pp. 115-121, Mar. 1988.

[4] M. H. Freilich and D. B. Chelton, "Wavenumber spectra of Pacific winds measured by the Seasat scatterometer," $J$. Phys. Oceanogr., vol 16, no. 4, pp. 741-757, Apr. 1986.

[5] W. L. Grantham et al., "Performance evaluation of an operational spaceborne scatterometer," IEEE Trans. Geosci. Remote Sensing, vol. GE-20, pp. 250-254, July 1982.

[6] D. G. Long, C.-Y. Chi, and F. K. Li, "The design of an onboard digital Doppler processor for a spaceborne scatterometer,' IEEE Trans. Geosci. Remote Sensing, vol. 26, pp. 869-878, Nov. 1988.

[7] D. Long, R. S. Dunbar, S. Shaffer, M. H. Freilich, and S. V. Hsiao, "Results of a study on polarization mix selection for the NSCAT scatterometer," in Proc. Int. Geosci. Remote Sensing Symp., July 1989, pp. 1454-1457.

[8] K. V. Mardia, Statistics of Directional Data. New York: Academic, 1972.

[9] B. D. Martin, M. H. Freilich, F. K. Li, and P. S. Callahan, "An overview of the NSCAT/N-ROSS program,"' in Proc. Workshop ERS-I Wind and Wave Calibration, June 2-6, 1986, pp. 143-149 (ESA Sp-262), 1986.

[10] L. C. Schroeder et al., "Removal of ambiguous wind directions for a $K u$-band wind scatterometer using measurements at three different azimuth angles," IEEE Trans. Geosci. Remote Sensing, vol. GE-23, pp. 91-100, Mar. 1985.

[11] M. G. Wurtele, P. M. Woiceshyn, S. Peteherych, M. Borowsky, and W. S. Appleby, "Wind direction alias removal studies of Seasat scatterometer-derived wind fields," J. Geophys. Res., vol. 87, no. C5, pp. 3365-3377, Apr. 1982. 


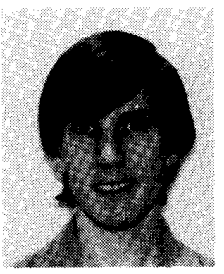

Scott J. Shaffer (S'84-M'86) received the B.S. degree in electrical engineering from the Uni versity of California at Davis in 1984, and the M.S. degree, also in electrical engineering, from the University of California at Los Angeles in 1986

Since then he has worked at the Jet Propulsion Laboratory as a Systems Engineer on both airborne and spaceborne radars. He worked on the NASA Scatterometer project for two years, contributing to the design and evaluation of the ambiguity removal algorithm. Currently, he is conducting performance tests of software which controls the Magellan synthetic aperture radar now mapping Venus.

R. Scott Dunbar was born in New York State in 1954. He received the B.S. degree in physics and astronomy from the State University of New York at Albany in 1976, and the Ph.D. degree in physics from Princeton University in 1980.

After two years as a National Research Council Resident Research Associate at the Jet Propulsion Laboratory (JPL), he joined JPL in 1983 as a Member of the Technical Staff. Since 1985 he has been involved in algorithm development for the NASA Scatterometer groundprocessing system.
S. Vincent Hsiao received the B.S. and M.S. degrees from the National Taiwan University in 1970 and 1972 , respectively, and the Ph.D. degree from the University of Florida in 1978.

He has been with the Jet Propulsion Laboratory since 1976, where his current technical interests are in the area of remote sensing.

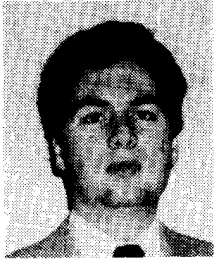

David G. Long (S'80-M'89) received the B.S. and M.S. degrees in electrical engineering from Brigham Young University, Provo, UT, in 1982 and 1983, respectively, and the Ph.D. degree in electrical engineering from the University of Southern California in Los Angeles in 1989.

He has worked for the Jet Propulsion Laboratory as a Radar Systems Engineer since 1983. $\mathrm{He}$ is currently the Project Engineer for the NASA Scatterometer (NSCAT) project and the Experiment Manager for the EOS SCANSCAT. He is responsible for the high-level design, analysis, and technical management of these projects to ensure that the projects meet all mission requirements. His responsibilities include overseeing instrument design and fabrication, algorithm and coding for the ground processing system, mission operations, and data analysis. His technical responsibilities include system performance analysis, high-level design, development and maintenance of system requirements, and supervision of the Systems Engineering staff. He is also a Group Leader, supervising a staff of system engineers working on a number of JPL flight projects. His research interests include computer graphics, speech and signal processing, estimation theory, radar, and mesoscale atmospheric dynamics.

Dr. Long has received the NASA Award of Achievement several times. He is a member of Tau Beta Pi. 